\title{
Corrigendum: Room-temperature chiral magnetic skyrmions in ultrathin magnetic nanostructures
}

Olivier Boulle, Jan Vogel, Hongxin Yang, Stefania Pizzini, Dayane de Souza Chaves, Andrea Locatelli, Tevfik Onur Menteș, Alessandro Sala, Liliana D. Buda-Prejbeanu, Olivier Klein, Mohamed Belmeguenai, Yves Roussigné, Andrey Stashkevich, Salim Mourad Chérif, Lucia Aballe, Michael Foerster, Mairbek Chshiev, Stéphane Auffret, Ioan Mihai Miron and Gilles Gaudin

Nature Nanotechnology 11, 449-454 (2016); published online 25 January 2016; corrected after print 18 July 2017

In the version of this Article originally published, the Dzyaloshinskii-Moriya interaction parameter, $D$, should have been multiplied by $\sqrt{ } 3$. The vertical scale of Fig. 2 has been updated accordingly as has the following sentence concerning $D$ values: "For 5 monolayers (ML) of Co, equivalent to a total Co thickness of $1 \mathrm{~nm}$, the ab initio calculations predict $D=4.0 \mathrm{~mJ} \mathrm{~m}^{-2}$ whereas a lower value $D=2.6 \mathrm{~mJ} \mathrm{~m}^{-2}$ is predicted for a Pt/Co/vacuum structure." Additionally the Methods and Supplementary Information have been updated to correct the experimental multilayer composition to: “ $\mathrm{Ta}(3) / \mathrm{Pt}(3) / \mathrm{Co}(0.5-1) / \mathrm{MgO}_{x} / \mathrm{Ta}(2)$ (thickness in $\mathrm{nm}$ )”. 